\title{
Antibacterial efficacy of prophylactic besifloxacin $0.6 \%$ and moxifloxacin $0.5 \%$ in patients undergoing cataract surgery
}

This article was published in the following Dove Press journal:

Clinical Ophthalmology

13 May 2015

Number of times this article has been viewed

Frank A Bucci Jr'

Ruth E Evans'

Loretta M Amico'

Timothy W Morris ${ }^{2}$

Angel T Fluet'

Christine M Sanfilippo 3

Heleen H DeCory ${ }^{3}$

Timothy L Comstock ${ }^{3}$

'Bucci Laser Vision Institute, WilkesBarre, PA, USA; ${ }^{2}$ Microbiology and Sterilization Sciences, Bausch and Lomb, Rochester, NY, USA; ${ }^{3}$ Medical Affairs, Bausch and Lomb, Rochester, NY, USA
Correspondence: Frank A Bucci Jr Bucci Laser Vision Institute, I58 Wilkes-Barre Township Boulevard, Wilkes-Barre, PA 18702, USA

$\mathrm{Tel}+\mathrm{I} 5708255949$

Fax +I 5708252645

Email buccivision@aol.com
Background: The purpose of this study was to investigate the ocular bacterial flora in patients scheduled to undergo cataract surgery and compare the antibacterial effects of besifloxacin ophthalmic suspension $0.6 \%$ and moxifloxacin ophthalmic solution $0.5 \%$ in these patients.

Methods: This was a prospective, randomized, laboratory-masked clinical trial. Patients received besifloxacin or moxifloxacin "quater in die" or QID (four times a day) for 3 days before cataract surgery in the surgical eye and 1 hour before surgery in the nonsurgical fellow eye. Conjunctival and eyelid swabs were obtained from both eyes at baseline and after treatment, on the day of surgery (Visit 2). Swabs were processed for bacterial colony counts (in terms of colony-forming units) and species identification. In vitro antibiotic susceptibilities of isolates were determined using Clinical and Laboratory Standards Institute breakpoints.

Results: Fifty-nine patients ( $n=28$ besifloxacin, $n=31$ moxifloxacin) completed the study. The majority $(73 \%)$ of conjunctival samples were culture negative at baseline. The most frequent isolates were coagulase-negative staphylococci (CoNS, 89\%), specifically Staphylococcus epidermidis (72\%). Both fluoroquinolones reduced the lid CFU values when administered QID for 3 days $(P \leq 0.019)$, but only besifloxacin reduced the lid CFU estimate 1 hour following instillation of a single drop $(P=0.039)$. Fewer besifloxacin-treated eyes had lids that were culture positive for CoNS at Visit 2 compared with moxifloxacin-treated eyes regardless of dosing regimen $(P \leq 0.03)$. The minimum inhibitory concentration $\left(\mathrm{MIC}_{90}\right)$ of besifloxacin against methicillin-resistant $S$. epidermidis (MRSE) was eightfold lower than that of moxifloxacin.

Conclusion: Besifloxacin appeared more effective in reducing bacterial counts on eyelids of patients undergoing cataract surgery, with significant reductions as early as 1 hour postdose, compared with moxifloxacin. Besifloxacin was more active in vitro against MRSE.

Keywords: besifloxacin, moxifloxacin, prophylaxis, cataract patients, coagulase-negative staphylococci

\section{Introduction}

Bacterial endophthalmitis is a rare but potentially vision-threatening complication of cataract surgery. ${ }^{1}$ The most common causes of bacterial endophthalmitis are grampositive organisms, often originating from resident bacterial flora on the conjunctiva and/or eyelids, which penetrate the eye during surgery or following surgery through the incision. ${ }^{2,3}$ Accordingly, the most frequently isolated organisms from cultureproven cases of postoperative endophthalmitis are coagulase-negative staphylococci (CoNS), most notably Staphylococcus epidermidis, ${ }^{3-9}$ also the most prevalent organism of the ocular flora in these patients. ${ }^{10-12}$ While the previously published European Society of Cataract and Refractive Surgeons (ESCRS) study established the efficacy of intraoperative, intracameral antibiotics in the prophylaxis against infection following 
cataract surgery, ${ }^{13}$ topical perioperative antibiotics, most often fluoroquinolones, remain a standard of care for prophylaxis against endophthalmitis in the US. ${ }^{14-17}$ However, cases of endophthalmitis due to fluoroquinolone-resistant isolates have begun to emerge. ${ }^{18-21}$

Besifloxacin, a chlorinated fluoroquinolone, is the latestgeneration topical fluoroquinolone with broad-spectrum antibacterial activity, including activity against drug-resistant staphylococci, ${ }^{22-26}$ comparable to that of vancomycin..$^{25,26}$ Besifloxacin was developed for ophthalmic use only and has been reported to have potent and balanced activity against DNA gyrase and topoisomerase IV. Both of these features have been suggested to reduce the potential for resistance development. ${ }^{27}$ Approved by the US Food and Drug Administration for the treatment of bacterial conjunctivitis, like other topical fluoroquinolone formulations, besifloxacin ophthalmic suspension $0.6 \%$ (Besivance $^{\circledR}$; Bausch and Lomb, Tampa, FL, USA) has been used perioperatively for prophylaxis against endophthalmitis.

The objective of this study was to investigate the ocular bacterial flora in patients scheduled to undergo cataract surgery and to compare the antibacterial efficacy of besifloxacin ophthalmic suspension $0.6 \%$ with that of moxifloxacin ophthalmic solution $0.5 \%\left(\right.$ Vigamox $^{\circledR}$, Alcon Laboratories, Inc, Fort Worth, TX, USA) in decreasing the bacterial load on lid margins and conjunctiva of these patients following two different preoperative dosing regimens. Moxifloxacin was chosen as a comparator because it is often used for prophylaxis in the surgical setting and has been described as a preferred option. ${ }^{15,28,29}$ We also determined the in vitro susceptibility of ocular bacterial isolates from these patients to besifloxacin, moxifloxacin, and a broad range of other antibacterials.

\section{Materials and methods Study design}

This randomized, laboratory-masked, active comparator, parallel group study (ClinicalTrials.gov identifier: NCT01296542) was conducted at a single-center, the Bucci Laser Vision Institute, between May 2011 and October 2012. The study was conducted in accordance with the current International Committee on Harmonization Guideline on Good Clinical Practices, as well as the Declaration of Helsinki, and was approved by a review board (RCRC Independent Review Board, Austin, TX, USA). Before enrollment, all patients gave written informed consent.

Eligible subjects were 18 years of age and older scheduled to undergo phacoemulsification with intraocular lens implantation for the treatment of cataract. Subjects had to be physically able to instill eye drops or have an appropriate person available to assist in administration of eye drops four times a day. Subjects had to meet the American Society of Anesthesiology physical status I, II, or III and be medically cleared for surgery. Subjects were excluded if they had a known allergy or contraindication to the test medication(s) or their components, or if they had any abnormality or significant illness in the eye that in the investigator's opinion could affect the subject's health or the study parameters. Subjects were also excluded if they had an active ocular infection (bacterial, viral, or fungal) or positive history of ocular herpetic infection; if they used fluoroquinolone or nonfluoroquinolone anti-infective agents (systemic or topical) within 1 week of Visit 1 (baseline) or were expected to use nonstudy fluoroquinolones or nonfluoroquinolones at any time during the study; used contact lenses for 1 week before the study or were expected to use contact lenses at any time during the study; or received an experimental drug or used an experimental medical device within 21 days before the planned start of treatment.

\section{Treatments and sample collection}

Subjects were screened for enrollment at Visit 1 only after providing written informed consent, at which time demographic data, medical history, and medication history were recorded. An undilated slit-lamp biomicroscopy examination was performed to examine both eyes for tissue abnormalities or other ocular disease. To avoid bias, a computer-generated list of randomized numbers was used in the assignment of eligible subjects to treatment with besifloxacin ophthalmic suspension $0.6 \%$ or moxifloxacin ophthalmic solution $0.5 \%$ in a 1:1 ratio. Subjects self-administered one drop of study drug into the surgical eye "quater in die" or QID (four times a day; 8 am, 12 pm, 4 pm, and 8 pm) for 3 days before surgery and recorded each dose administered in a diary. On the day of surgery, subjects received a single drop of the assigned study medication in their nonsurgical eye 1 hour before surgery. Subjects who reported missing any dose of study medication were discontinued from the study.

Bacterial samples were obtained by study personnel from the superior lid lash margin and inferior tarsal conjunctiva of both eyes using a sterile swab before beginning the 3-day QID treatment (Visit 1/baseline) and then again 1 hour following dosing of the nonsurgical eye on the day of surgery (Visit 2). At each visit, special care was taken when obtaining conjunctival samples, avoiding contact with the eyelids. Samples of Visit 2 were collected just before surgery, as well 
as before treatment with topical anesthetics, dilating drops, or antiseptics (ie, Betadine). If subjects had all conjunctival and lid culture samples collected, they were considered to have completed the study and were subsequently referred to as "patients."

Swabs were inserted into transport medium (20\% glycerol in phosphate-buffered saline), immediately refrigerated $\left(2^{\circ} \mathrm{C}-8^{\circ} \mathrm{C}\right)$ and shipped under refrigerated conditions using frozen gel packs the same day as collection (or if collected on the weekend, on Monday) by overnight delivery to a central laboratory (Covance Central Laboratory Services, Indianapolis, IN, USA) for quantitative bacteriological analysis. Briefly, serial dilutions of test samples were inoculated onto bacteriological media, and the resulting colonies were enumerated and speciated by standard biochemical and/or molecular identification methods (genetic ribotyping, 16S ribosomal RNA gene sequencing, and/or automated biochemical testing panels). Minimum inhibitory concentration (MIC) testing was performed on all isolates by broth microdilution for besifloxacin, moxifloxacin, and a panel of comparator antibacterials following the recommended procedures of the Clinical and Laboratory Standards Institute (CLSI). ${ }^{30}$ Comparator antibacterials included a representative betalactam antibiotic (oxacillin), older fluoroquinolones (gatifloxacin, ciprofloxacin, and levofloxacin), macrolides (azithromycin, erythromycin), an aminoglycoside (tobramycin), trimethoprim, chloramphenicol, and a glycopeptide (vancomycin). The $\mathrm{MIC}_{50}$ and $\mathrm{MIC}_{90}$ (MIC for 50\% and 90\% of isolates, respectively) were calculated for any prevalent species, and susceptibility/nonsusceptibility of isolates was interpreted using CLSI breakpoints. ${ }^{31}$

\section{Safety}

Throughout the study, ocular and nonocular adverse events (reported, elicited, and observed) were to be recorded and coded using the Medical Dictionary for Regulatory Activities. For each adverse event, the percentage of patients who experienced at least one occurrence of the given event was to be summarized by treatment group.

\section{Statistical analysis}

A total of 60 patients $(n=30$ per treatment arm) were planned to be enrolled. Analyses were performed with descriptive statistics on all patients who completed both preoperative visits. Eyes of patients completing both study visits were included in the evaluation of the spectrum of ocular surface flora and antimicrobial susceptibility. Changes in colony-forming units per milliliter $(\mathrm{CFU} / \mathrm{mL})$ were evaluated using the
Student's $t$-test for paired samples. Two-tailed $P$-values were calculated, and significance was set at 0.05 . The Pearson's chi-square test was used to evaluate differences in proportions. For individual isolates, posttreatment characterization of changes in CFU/mL values as increased or decreased relative to baseline were based on a threshold of a $>4$-fold increase or decrease. Posttreatment isolates in which the change in $\mathrm{CFU} / \mathrm{mL}$ estimate was $<4$-fold relative to baseline were considered unchanged.

\section{Results}

\section{Patient demographics}

A total of 67 patients were randomized ( $n=33$ besifloxacin, $\mathrm{n}=34$ moxifloxacin) and 59 patients completed the study ( $n=28$ besifloxacin, $n=31$ moxifloxacin). Eight subjects discontinued the study ( $n=5$ besifloxacin, $n=3$ moxifloxacin). Reasons for study discontinuation included subject noncompliance, sample collection error, and cancellation of surgery. The mean ( \pm standard deviation) age was 68.1 $( \pm 8.6)$ years, $45.8 \%$ of patients were female, and all patients were Caucasian. Patients' medical histories and comorbid conditions were typical of a population in the seventh decade of life.

\section{Baseline bacteria and in vitro antibiotic susceptibility}

Only $27 \%$ of conjunctival samples were culture positive at baseline compared with $87 \%$ of lid margin samples. Lid margins harbored a greater quantity and variety of bacterial species at baseline (pretreatment/Visit 1) compared to the conjunctiva. The mean ( \pm standard error of the mean [SEM]) $\mathrm{CFU} / \mathrm{mL}$ estimate of all samples was 2,671 $( \pm 566) \mathrm{CFU} / \mathrm{mL}$ for lid cultures compared with $38( \pm 20) \mathrm{CFU} / \mathrm{mL}$ for conjunctival cultures and were comparable between surgical eyes and nonsurgical fellow eyes.

Table 1 presents the distribution of bacteria at baseline (pretreatment) by species and origin (lid margins, conjunctiva) in patients who completed the study. A total of 178 isolates were identified, representing 17 unique species. The distribution of bacterial species was similar between surgical eyes and nonsurgical fellow eyes. All but four isolates were gram-positive species, and the predominant organisms were CoNS, representing 89\% (158/178) of all bacterial isolates identified. The single most prevalent species was S. epidermidis, accounting for $81 \%$ (128/158) of CoNS isolates and $72 \%(128 / 178)$ of all bacterial isolates. Several lid cultures $(n=17)$ harbored two or more CoNS species.

All patients were asymptomatic for infection at baseline. Among the few conjunctival samples that were culture positive 
Table I Distribution of bacterial isolates at baseline from lid margins and conjunctiva of patients undergoing cataract surgery

\begin{tabular}{|c|c|c|c|c|c|}
\hline & \multicolumn{4}{|c|}{ Number of isolates } & \multirow[t]{3}{*}{ Total (\%) } \\
\hline & \multicolumn{2}{|l|}{ Lid margin } & \multicolumn{2}{|l|}{ Conjunctiva } & \\
\hline & Surgical eye & Fellow eye & Surgical eye & Fellow eye & \\
\hline \multicolumn{6}{|l|}{ Gram-positive bacteria } \\
\hline \multicolumn{6}{|l|}{ CoNS } \\
\hline Staphylococcus epidermidis & 50 & 55 & 12 & 11 & I28 (7I.9) \\
\hline Staphylococcus capitis & 5 & 5 & & & $10(5.6)$ \\
\hline Staphylococcus lugdunensis & 4 & 4 & & & $8(4.5)$ \\
\hline Staphylococcus warneri & 2 & 3 & I & 2 & $8(4.5)$ \\
\hline Staphylococcus hominis & 1 & I & & & $2(1.1)$ \\
\hline Staphylococcus haemolyticus & I & & & & $\mathrm{I}(0.6)$ \\
\hline Staphylococcus pasteuri & & I & & & $\mathrm{I}(0.6)$ \\
\hline Staphylococcus aureus & 2 & 2 & 1 & 2 & $7(3.9)$ \\
\hline Coryneform bacteria ${ }^{\mathrm{b}}$ & I & 2 & I & & $4(2.2)$ \\
\hline Kocuria varians & I & I & & & $2(1.1)$ \\
\hline Rothia mucilaginosa & & & 1 & 1 & $2(1.1)$ \\
\hline Micrococcus luteus & & I & & & $\mathrm{I}(0.6)$ \\
\hline Total & 67 & 75 & 16 & 16 & $174(97.8)$ \\
\hline \multicolumn{6}{|l|}{ Gram-negative bacteria $^{c}$} \\
\hline Acinetobacter calcoaceticus & 1 & & 1 & & $2(1.1)$ \\
\hline Stenotrophomonas maltophilia & I & & I & & $2(I . I)$ \\
\hline Total & 2 & & 2 & & $4(2.2)$ \\
\hline
\end{tabular}

Notes: ${ }^{a}$ Percentage of all isolates identified. 'Includes Corynebacterium simulans, Corynebacterium pseudodiphtheriticum, CDC coryneform group G, and Dermabacter hominis. 'All gram-negative isolates were cultured from the surgical eye of a single patient.

Abbreviations: CDC, Centers for Disease Control and Prevention; CoNS, coagulase-negative staphylococci.

at baseline, colony counts for any individual species were below the threshold considered to be pathogenic in bacterial conjunctivitis as defined on the Cagle list and modified by Leibowitz. ${ }^{32}$ The only exceptions were the gram-negative isolates, Acinetobacter calcoaceticus and Stenotrophomonas maltophilia (cultured from both the conjunctival and lid margin sample of one eye), which were present at $>1 \mathrm{CFU} / \mathrm{mL}$, which is considered the pathogenic threshold for those species.

The in vitro antibiotic susceptibility of CoNS isolated at baseline is shown in Table 2. CoNS isolates were least susceptible to the macrolides ( $68 \%$ ), followed by oxacillin $(82 \%)$, the latter being a surrogate for methicillin resistance. Susceptibility was high for tobramycin (99\%), chloramphenicol (98\%), and vancomycin (100\%), but slightly lower for fluoroquinolones with established breakpoints (88\%-89\%). Because besifloxacin was developed for topical ophthalmic use, a CLSI breakpoint is not available to determine susceptibility to besifloxacin. However, using breakpoints for moxifloxacin as a surrogate, the susceptibility of CoNS to besifloxacin was predicted to be higher (99\%) than that of CoNS to other fluoroquinolones. Of the fluoroquinolones tested, besifloxacin had the lowest $\mathrm{MIC}_{90}$ against CoNS $(0.5 \mu \mathrm{g} / \mathrm{mL})$, lower than that of vancomycin $(2 \mu \mathrm{g} / \mathrm{mL})$. Nearly identical susceptibility trends were noted for the subgroup of S. epidermidis isolates (data not shown). S. aureus isolates were susceptible to all the antibiotics tested, with the exception of macrolides (only $43 \%$ susceptibility to azithromycin, $29 \%$ susceptibility to erythromycin, data not shown). No notable resistance trends were observed among the remaining gram-positive isolates.

Table 2 In vitro antibiotic susceptibility of CoNS recovered at baseline from lid margins and conjunctiva of cataract patients

\begin{tabular}{|c|c|c|c|c|}
\hline \multirow[t]{2}{*}{ Drugs evaluated } & \multicolumn{3}{|l|}{ MIC $(\mu \mathrm{g} / \mathrm{mL})$} & \multirow[t]{2}{*}{$\%$ susceptible $^{\mathrm{a}}$} \\
\hline & Range & $\mathrm{MIC}_{50}$ & $\mathrm{MIC}_{90}$ & \\
\hline \multicolumn{5}{|l|}{ All CoNS $(\mathrm{N}=158)$} \\
\hline Oxacillin & $<0.06->128$ & 0.12 & 2 & 82 \\
\hline Besifloxacin & $0.015-4$ & 0.06 & 0.5 & NA (99) ${ }^{c}$ \\
\hline Moxifloxacin & $0.03-32$ & 0.12 & I & 89 \\
\hline Gatifloxacin & $0.06-32$ & 0.12 & 2 & 88 \\
\hline Ciprofloxacin & $0.06-64$ & 0.25 & 4 & 89 \\
\hline Levofloxacin & $0.06-256$ & 0.25 & 4 & 89 \\
\hline Azithromycin & $<0.008->256$ & 2 & $>256$ & 68 \\
\hline Erythromycin & $<0.008->32$ & 0.5 & $>32$ & 68 \\
\hline Tobramycin & $<0.06-64$ & 0.12 & 0.25 & 99 \\
\hline Trimethoprim & $<0.03->64$ & 2 & 16 & 88 \\
\hline Chloramphenicol & $1-16$ & 4 & 8 & 98 \\
\hline Vancomycin & $0.5-4$ & 2 & 2 & 100 \\
\hline
\end{tabular}

Notes: a Determination of percentage susceptibility based on Clinical and Laboratory Standards Institute breakpoints for the respective CoNS species listed above. IIncludes Staphylococcus capitis $(n=10)$, Staphylococcus epidermidis $(n=128)$, Staphylococcus haemolyticus $(n=l)$, Staphylococcus hominis $(n=2)$, Staphylococcus lugdunensis $(n=8)$, Staphylococcus pasteuri $(\mathrm{n}=\mathrm{l})$, and Staphylococcus warneri $(\mathrm{n}=8) .{ }^{\circ}$ There are no susceptibility breakpoints for besifloxacin; percentage susceptible to besifloxacin was calculated using moxifloxacin breakpoints.

Abbreviations: CoNS, coagulase-negative staphylococci; MIC, minimum inhibitory concentration; $\mathrm{MIC}_{50}$, minimum inhibitory concentration for $50 \%$ of isolates; $\mathrm{MIC}_{90}$, minimum inhibitory concentration for $90 \%$ of isolates; NA, breakpoint for determination of susceptibility not available. 
As indicated above, 18\% (28/158) of CoNS, including 27 S. epidermidis isolates and $1 \mathrm{~S}$. hominis isolate, were characterized as methicillin-resistant species based on oxacillin testing. As summarized in Table 3, susceptibilities among the methicillin-resistant S. epidermidis (MRSE) isolates remained high for chloramphenicol (96\%) and vancomycin (100\%) but were much lower for tobramycin (59\%). None of the MRSE isolates were susceptible to the macrolides, and only a third of these were susceptible to older fluoroquinolones. In contrast, nearly all MRSE (93\%) isolates were predicted to be susceptible to besifloxacin when using the breakpoint for moxifloxacin as a surrogate. The $\mathrm{MIC}_{90}$ of besifloxacin against MRSE was eightfold lower than that of moxifloxacin and 8- to 128 -fold lower than that of other fluoroquinolones.

\section{Antibacterial efficacy of prophylactic besifloxacin and moxifloxacin treatment}

As indicated above, few conjunctival samples were culture positive at baseline. Both fluoroquinolones appeared to decrease mean conjunctival colony counts $(\mathrm{CFU} / \mathrm{mL})$ in surgical and nonsurgical fellow eyes (data not shown), but the changes from baseline did not reach statistical significance $(P \geq 0.129)$ due to the limited number of culturepositive samples and variability in those samples at baseline. Conjunctival samples were not evaluated further.

Both fluoroquinolones reduced overall colony counts on lid margins when administered QID for 3 days (surgical

Table 3 In vitro antibiotic susceptibility of MRSE recovered at baseline from lid margins and conjunctiva of cataract patients

\begin{tabular}{lllll}
\hline Drugs evaluated & \multicolumn{2}{l}{ MIC $(\mu \mathrm{g} / \mathrm{mL})$} & \% susceptible $^{\mathbf{a}}$ \\
\cline { 2 - 4 } & Range & MIC $_{50}$ & MIC $_{90}$ & \\
\hline MRSE (N=27) & & & & \\
Besifloxacin & $0.06-4$ & 0.5 & 0.5 & NA $(93)^{\mathrm{b}}$ \\
Moxifloxacin & $0.12-32$ & 2 & 4 & 33 \\
Gatifloxacin & $0.25-32$ & 2 & 4 & 33 \\
Ciprofloxacin & $0.5-64$ & 8 & 64 & 33 \\
Levofloxacin & $0.25-256$ & 8 & 16 & 33 \\
Azithromycin & $128->256$ & $>256$ & $>256$ & 0 \\
Erythromycin & $32->32$ & $>32$ & $>32$ & 0 \\
Tobramycin & $0.25-64$ & 0.25 & 32 & 59 \\
Trimethoprim & $2->64$ & 4 & $>64$ & 63 \\
Chloramphenicol & $8-16$ & 8 & 8 & 96 \\
Vancomycin & $2-4$ & 2 & 2 & 100 \\
\hline
\end{tabular}

Notes: a Determination of percentage susceptibility based on Clinical and Laboratory Standards Institute breakpoints for Staphylococcus epidermidis. ${ }^{\mathrm{t}}$ There are no susceptibility breakpoints for besifloxacin; percentage susceptible to besifloxacin was calculated using moxifloxacin breakpoint of $0.5 \mu \mathrm{g} / \mathrm{mL}$.

Abbreviations: MRSE, methicillin-resistant $S$. epidermidis; MIC, minimum inhibitory concentration; $\mathrm{MIC}_{50}$, minimum inhibitory concentration for $50 \%$ of isolates; $\mathrm{MIC}_{90}$, minimum inhibitory concentration for $90 \%$ of isolates; NA, breakpoint for determination of susceptibility not available. eyes). Mean ( \pm SEM) colony counts on lids decreased from $4,955( \pm 1,948) \mathrm{CFU} / \mathrm{mL}$ at baseline to $31( \pm 9) \mathrm{CFU} / \mathrm{mL}$ at Visit 2 in besifloxacin-treated eyes $(P=0.018)$ and from 1,122 $( \pm 366) \mathrm{CFU} / \mathrm{mL}$ to $200( \pm 52) \mathrm{CFU} / \mathrm{mL}$ in moxifloxacintreated eyes $(P=0.019)$. In contrast, only besifloxacin reduced the colony counts within 1 hour of instilling a single topical instillation to nonsurgical eyes. The mean ( \pm SEM) colony count decreased from $3,041( \pm 1,051) \mathrm{CFU} / \mathrm{mL}$ at baseline to $538[ \pm 364] \mathrm{CFU} / \mathrm{mL} 1$ hour following instillation of a single drop of besifloxacin $(P=0.039)$ but was not changed 1 hour following treatment with moxifloxacin $(1,793[ \pm 560]$ $\mathrm{CFU} / \mathrm{mL}$ at baseline to 2,201 [ \pm 919$] \mathrm{CFU} / \mathrm{mL} ; P=0.530$ ). As expected, given the decrease in colony counts observed, the proportion of surgical eyes with lid margins that were culture negative increased from $17.9 \%(5 / 28)$ at baseline to $42.8 \%$ $(12 / 28)$ at Visit 2 with besifloxacin treatment $(P=0.04)$ and from $16.1 \%(5 / 31)$ at baseline to $19.4 \%(6 / 31)$ at Visit 2 with moxifloxacin treatment $(P=0.74)$. Among nonsurgical fellow eyes, the proportion of culture-negative lid margins increased from $10.7 \%(3 / 28)$ at baseline to $53.6 \%(15 / 28)$ with besifloxacin treatment $(P<0.001)$ but decreased from $6.6 \%(2 / 30)$ at baseline to $0 \%(0 / 31)$ with moxifloxacin treatment $(P=0.14)$, with a statistical difference between treatments at Visit $2(P<0.001$; Table 4$)$.

Figure 1 presents the reductions in mean ( \pm SEM) colony counts for CoNS isolates. In surgical eyes treated QID for 3 days, mean ( \pm SEM) colony counts for CoNS from lids decreased from 4,931 $( \pm 1,950) \mathrm{CFU} / \mathrm{mL}$ to $28( \pm 9) \mathrm{CFU} / \mathrm{mL}$ following treatment with besifloxacin $(P=0.018)$ and from $1,090( \pm 368) \mathrm{CFU} / \mathrm{mL}$ to $126( \pm 23) \mathrm{CFU} / \mathrm{mL}$ following treatment with moxifloxacin $(P=0.014)$. As was the case for overall colony counts, the mean colony count for CoNS found on the lids of nonsurgical fellow eyes decreased from 3,036 $( \pm 1,051) \mathrm{CFU} / \mathrm{mL}$ to 537 [ \pm 364$] \mathrm{CFU} / \mathrm{mL} 1$ hour following instillation of a single drop of besifloxacin $(P=0.039)$ but was not significantly changed 1 hour following instillation of a single drop of moxifloxacin $(1,411[ \pm 361] \mathrm{CFU} / \mathrm{mL}$ at baseline to $1,922[ \pm 889] \mathrm{CFU} / \mathrm{mL}$ posttreatment; $P=0.542$ ). Consistent with these findings, the proportion of lid margins that were culture negative for CoNS following 3 days of QID treatment increased from $17.9 \%(5 / 28)$ to $50.0 \%$ $(14 / 28)$ with besifloxacin $(P=0.01)$ and from $19.4 \%(6 / 31)$ to $22.6 \%(7 / 31)$ with moxifloxacin $(P=0.76)$. However, while the proportion of lids that were culture negative 1 hour after instillation of a single drop also increased from $10.7 \%$ $(3 / 28)$ to $53.6 \%(15 / 28)$ with besifloxacin $(P<0.001)$, the proportion decreased from $9.7 \%(3 / 31)$ to $0 \%(0 / 31) 1$ hour after instillation of a single drop of moxifloxacin $(P=0.08)$. 
Table 4 Proportion of eyes with culture-negative lid margins following antibacterial treatment with two dosing regimens

\begin{tabular}{llll}
\hline Antibacterials & Besifloxacin ophthalmic suspension, 0.6\% & Moxifloxacin ophthalmic solution, 0.5\% $^{P}$-value \\
\hline All species & & & 0.05 \\
QID, 3 days (surgical eye) & $12 / 28$ & $6 / 31$ & $<0.001$ \\
Single drop (nonsurgical eye) & $15 / 28$ & $0 / 31$ & \\
CoNS & & & 0.03 \\
QID, 3 days (surgical eye) & $14 / 28$ & $7 / 31$ & $<0.001$ \\
Single drop (nonsurgical eye) & $15 / 28$ & $0 / 31$ & \\
\hline
\end{tabular}

Notes: ${ }^{2}$ Difference between treatments, Pearson's chi-square analysis.

Abbreviations: CoNS, coagulase-negative staphylococci; QID, "quater in die” (four times a day).

The difference between treatments in the proportion of lid margins that were culture negative at Visit 2 was significant in favor of besifloxacin regardless of dosing regimen $(P \leq 0.03$; Table 4).

As indicated above, 21\% (27/128) of S. epidermidis were methicillin resistant. Among eyes with lid cultures positive for MRSE at baseline, the mean colony counts for MRSE decreased from 15,856 $( \pm 9,587) \mathrm{CFU} / \mathrm{mL}$ and $538( \pm 247)$ $\mathrm{CFU} / \mathrm{mL}$ at baseline to $0 \mathrm{CFU} / \mathrm{mL}$ and $65( \pm 53) \mathrm{CFU} / \mathrm{mL}$ following treatment with besifloxacin and moxifloxacin, respectively, administered QID for 3 days; and from 7,855 $( \pm 4,099) \mathrm{CFU} / \mathrm{mL}$ and $1,094( \pm 579) \mathrm{CFU} / \mathrm{mL}$ at baseline to $544( \pm 520) \mathrm{CFU} / \mathrm{mL}$ and $213( \pm 98) \mathrm{CFU} / \mathrm{mL} 1$ hour following treatment with besifloxacin and moxifloxacin, respectively, administered as a single drop. The changes from baseline did not reach statistical significance, presumably due to the limited number of eyes with MRSE at baseline $(P \geq 0.0829)$.

\section{Bacteria present posttreatment}

Despite the significant reductions observed for mean colony counts on lid margins, analysis of bacterial isolates posttreatment showed that several eyes had CoNS isolates persisting posttreatment. Specifically, $20 \mathrm{CoNS}$ isolates were recovered from lid margins of besifloxacin-treated eyes ( $n=10$ each from surgical and nonsurgical fellow eyes), and 48 CoNS isolates were obtained from lid margins of moxifloxacin-treated eyes ( $n=20$ from surgical eyes, $n=28$ from nonsurgical fellow eyes) in which the CoNS was the same species as that isolated at baseline. Although many of these isolates were present at reduced colony counts relative to baseline, there were some CoNS isolates present at unchanged $(n=10$ besifloxacin, $n=24$ moxifloxacin) or increased ( $\mathrm{n}=1$ besifloxacin, $n=7$ moxifloxacin) colony counts, with the latter most often cultured from lid margins of nonsurgical fellow eyes. Analysis of the MICs of besifloxacin or moxifloxacin for CoNS isolates present at Visit 2 at unchanged or increased colony counts revealed three $S$. epidermidis isolates from two patients treated with besifloxacin in which the MIC for besifloxacin was $\geq 1 \mu \mathrm{g} / \mathrm{mL}$. Two of these isolates were methicillin resistant. Among CoNS isolates present at Visit 2 at unchanged or increased colony counts obtained from moxifloxacin-treated eyes, there were ten S. epidermidis isolates

\section{Besifloxacin}

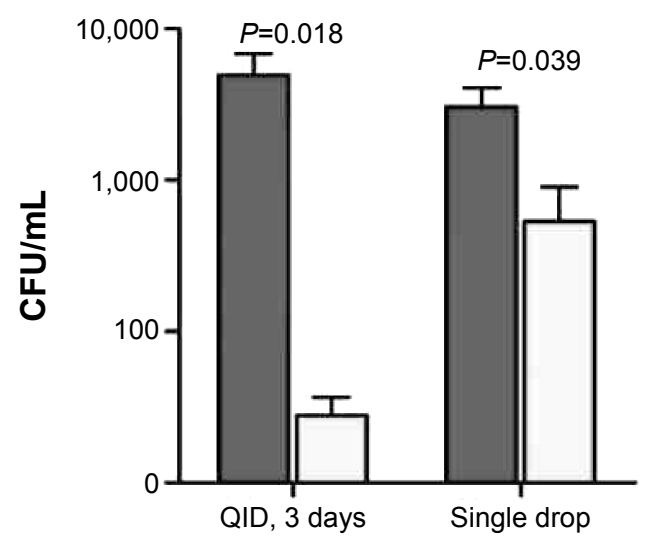

Moxifloxacin

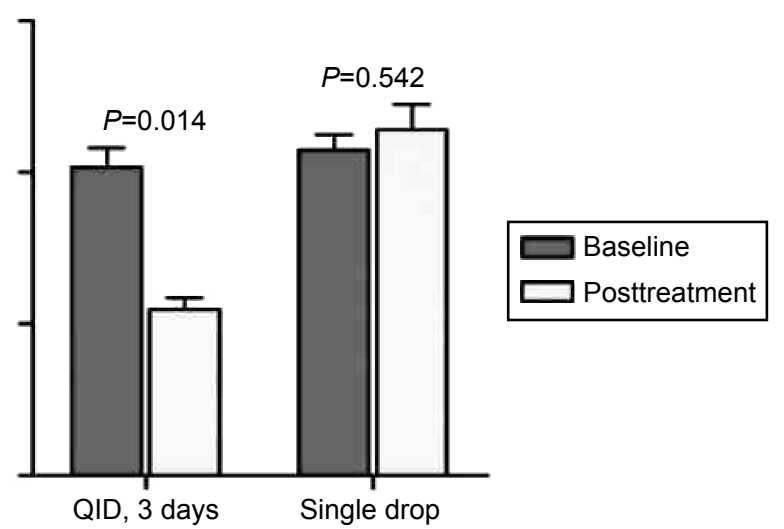

Figure I Mean CFU for CoNS on eyelid margins.

Notes: CFU for CoNS on eyelid margins at baseline (pretreatment) and following treatment with either besifloxacin or moxifloxacin administered QID for 3 days (surgical eye) or I hour after instilling a single drop (nonsurgical eye). Listed $P$-values compare baseline with posttreatment values of bacterial counts in CFU per milliliter. Abbreviations: CFU, colony-forming units; CoNS, coagulase-negative staphylococci; QID, "quater in die" (four times a day). 
(from eight patients) in which the MIC for moxifloxacin was $\geq 1 \mu \mathrm{g} / \mathrm{mL}$ (range: $1-64 \mu \mathrm{g} / \mathrm{mL}$ ) and thereby predictive of intermediate/full resistance to moxifloxacin. Of these ten isolates, seven were MRSE. Considering treatment outcomes for MRSE isolates only, among besifloxacin-treated eyes, there was only one baseline MRSE lid isolate that showed no change in colony counts following treatment (single drop instillation), whereas, among moxifloxacin-treated eyes, there were six baseline MRSE lid isolates with unchanged $(n=4)$ or increased $(n=2)$ colony counts at Visit 2.

Of the four eyes (three patients) with $S$. aureus isolates at baseline, the bacterium was absent at Visit 2 in two and was significantly reduced in colony counts in the other two. Of the two eyes with Rothia mucilaginosa isolates at baseline, the bacterium was absent from one, but present at low colony counts at Visit 2 in the other. There were no other gram-positive species present at baseline persisting at visit 2. The gram-negative isolates present at baseline were absent at Visit 2.

\section{Safety}

There were no adverse events, either ocular or nonocular, reported for any patient in either the besifloxacin or moxifloxacin treatment groups.

\section{Discussion}

In this study, besifloxacin ophthalmic suspension $0.6 \%$ and moxifloxacin ophthalmic solution $0.5 \%$ were both effective in decreasing bacterial colony counts on lid margins of patients scheduled to undergo cataract surgery following QID treatment for 3 days, but only besifloxacin was effective in decreasing colony counts 1 hour following instillation of a single drop. While the patients' conjunctival cultures were also analyzed before and after antibacterial treatment, there were too few eyes with positive conjunctival cultures at baseline and too great a variability in $\mathrm{CFU} / \mathrm{mL}$ estimates to evaluate the change in bacterial colony counts on the conjunctiva.

The results obtained in this study were generally consistent with and supportive of previous studies regarding reduction of risk from infection by ocular flora in the perioperative setting. Various studies have documented the efficacy of the fluoroquinolone class of antibiotics in reducing the ocular surface bacterial flora in patients scheduled to undergo cataract surgery when administered for 3 days before cataract surgery. ${ }^{33-35}$ However, published data for fluoroquinolones administered 1 hour before surgery have been less consistent,,$^{33,36,37}$ leading to suggestions that 1 hour may not allow adequate exposure time for some fluoroquinolones to reduce the bacterial load. ${ }^{33}$ Haas et $\mathrm{al}^{38,39}$ showed that besifloxacin was more rapidly bactericidal than moxifloxacin when tested in vitro against staphylococcal isolates, including methicillin-resistant strains. Specifically, besifloxacin led to a 3-log ( $\geq 1,000$-fold) kill within 1 hour, whereas moxifloxacin required $\geq 2$ hours to reach a 3-log kill. ${ }^{39}$ Until now, the clinical corollary of these data had not been tested. In addition, the besifloxacin formulation contains DuraSite, a mucoadhesive polyacrylic acid polymer designed to improve the retention time of a drug on the ocular surface. ${ }^{40-44}$ It is likely that the polyacrylic acid polymer in the besifloxacin formulation retained besifloxacin on the ocular surface longer, thereby augmenting besifloxacin's intrinsic bactericidal activity. The formulation of moxifloxacin tested in this investigation contains no vehicle components to increase surface contact time.

The most prevalent organisms isolated in this study were CoNS, and among these, $18 \%$ were found to be methicillin resistant at baseline, with all but one isolates being MRSE. Besifloxacin was substantially more potent than moxifloxacin in vitro against MRSE present at baseline, with $\mathrm{MIC}_{90}$ values that were eightfold lower than those of moxifloxacin. Utilizing the CLSI breakpoint for moxifloxacin, nearly all MRSE isolates were predicted to be susceptible to besifloxacin but only one-third were predicted to be susceptible to moxifloxacin. Although there were too few eyes with MRSE at baseline with too great a variability in colony counts to demonstrate a significant decrease in colony counts of MRSE with either besifloxacin or moxifloxacin treatment, it is notable that only one MRSE isolate was cultured from eyes treated with besifloxacin, whereas among moxifloxacintreated eyes, there were six lid MRSE isolates at baseline with unchanged or increased colony counts posttreatment. Elimination of MRSE and methicillin-resistant $S$. aureus (MRSA) from the ocular surface has been of increasing concern to cataract surgeons. In 2010, Olson et al ${ }^{10}$ reported that MRSE accounted for $47.1 \%$ of S. epidermidis isolates and MRSA for $29.5 \%$ of $S$. aureus isolates at the time of lid culture in cataract patients. Similarly, in studies of conjunctival flora of patients scheduled to undergo cataract surgery, MRSE accounted for $22.6 \%-54.0 \%$ of $S$. epidermidis isolates and MRSA for $27.3 \%-64.0 \%$ of $S$. aureus isolates. ${ }^{11,12}$ The rate of methicillin resistance in the current study was lower than that reported in these previous studies. However, based on results from in vitro data, it is possible that besifloxacin treatment may demonstrate an advantage over moxifloxacin treatment among cataract patients with a greater proportion of methicillin-resistant staphylococcal isolates. 
Perhaps more relevant, among moxifloxacin-treated eyes, there were ten S. epidermidis isolates persisting posttreatment, with MICs classified as moxifloxacin-resistant, compared to three $S$. epidermidis isolates persisting posttreatment in besifloxacin-treated eyes with an MIC $\geq 1 \mu \mathrm{g} / \mathrm{mL}$, the susceptibility breakpoint for moxifloxacin. One might speculate that, given its relatively recent introduction to the market, and with no systemic counterpart to provide exposure and induce resistance development, resistance to besifloxacin will emerge more slowly, although crossresistance from other fluoroquinolones is possible. In this study, $11 \%-12 \%$ of isolates at baseline were found to be resistant to ciprofloxacin, moxifloxacin, gatifloxacin, and levofloxacin. Sizable percentages of staphylococcal isolates resistant to these older fluoroquinolones have been identified in a number of recent endophthalmitis reports ${ }^{45-49}$ and appear to be on the rise, often in conjunction with methicillin resistance.

Several studies have examined the safety of besifloxacin ophthalmic suspension $0.6 \%$ compared with that of moxifloxacin ophthalmic solution $0.5 \%$ when used for prophylaxis against infections in cataract patients, ${ }^{50-52}$ laser-assisted in situ keratomileusis patients, ${ }^{52,53}$ and in patients undergoing photorefractive keratectomy. ${ }^{54}$ Results from these studies using preoperative and/or postoperative dosing regimens showed that both antibacterials were well tolerated, with no safety concerns and no differences between treatments in surgical outcomes (data not shown). Consistent with previously reported findings, there were no safety concerns in the current study; in fact, there were no adverse events recorded with either fluoroquinolone in our study.

Our study had two limitations. The first limitation was the lack of genetic testing of isolate pairs of the same species when present both at baseline and posttreatment in the same eye to confirm the persistence of a strain cultured at baseline versus the possibility of a new strain of the same species colonizing the eye at follow-up. In this regard, it is important to note that a number of eyes were colonized with a different CoNS species at Visit 2 compared to baseline (data not shown). To better evaluate the prophylactic effect of besifloxacin and moxifloxacin in our study, we chose to limit our analysis of bacterial isolates present posttreatment in individual eyes to those species that were identified in those eyes at baseline. The second limitation was that we did not control for the potential contribution of benzalkonium chloride (BAK) in eyes treated with besifloxacin ophthalmic suspension. The besifloxacin formulation contains $0.01 \%$ BAK in addition to besifloxacin and the mucoadhesive vehicle, DuraSite. BAK has been shown to have bactericidal properties of its own. ${ }^{39,55}$ It is therefore possible that the decrease in lid colony counts 1 hour following treatment with besifloxacin ophthalmic suspension may have been due, in part, to residual activity of BAK. Without a BAKfree formulation of besifloxacin, we cannot determine the degree of the contribution of residual BAK, if any, to the overall bacterial killing observed with the besifloxacin formulation.

In conclusion, the results of our study suggest that besifloxacin was more effective than moxifloxacin in reducing CoNS colony counts on lid margins and conjunctiva of patients undergoing cataract surgery. Treatment with besifloxacin led to more lid cultures with no growth posttreatment, and only besifloxacin was able to decrease the bacterial load 1 hour following a single drop instillation. Along with besifloxacin's in vitro potency against MRSE, the findings of this study provide compelling evidence to support the use of besifloxacin as part of a strategy to reduce the risk of bacterial endophthalmitis associated with cataract surgery.

\section{Acknowledgments}

The authors thank Churchill Communications (Maplewood, NJ, USA) for editorial assistance, which was supported by Bausch and Lomb.

\section{Disclosure}

FAB Jr discloses that he is a paid consultant for Bausch and Lomb and that this study was sponsored by an unrestricted research grant from Bausch and Lomb. TWM, CMS, HHD, and TLC disclose being employees of Bausch and Lomb during the conduct of this analysis. The other authors report no conflict of interest in this work.

\section{References}

1. Gower EW, Lindsley K, Nanji AA, Leyngold I, McDonnell PJ. Perioperative antibiotics for prevention of acute endophthalmitis after cataract surgery. Cochrane Database Syst Rev. 2013;7:CD006364.

2. Speaker MG, Milch FA, Shah MK, Eisner W, Kreiswirth BN. Role of external bacterial flora in the pathogenesis of acute postoperative endophthalmitis. Ophthalmology. 1991;98(5):639-650.

3. Mah FS, Davidson R, Holland EJ, et al; ASCRS Cornea Clinical Committee. Current knowledge about and recommendations for ocular methicillin-resistant Staphylococcus aureus. J Cataract Refract Surg. 2014;40(11):1894-1908.

4. Holland EJ, McDonald MB, Parekh JG, Sheppard JD. Antibiotic resistance in acute postoperative endophthalmitis. Ophthalmology. 2014; 121(11 suppl):S1-S9.

5. Benz MS, Scott IU, Flynn HW Jr, Unonius N, Miller D. Endophthalmitis isolates and antibiotic sensitivities; a 6-year review of culture-proven cases. Am J Ophthalmol. 2004;137(1):38-42.

6. Gentile RC, Shukla S, Shah M, et al. Microbiological spectrum and antibiotic sensitivity in endophthalmitis: a 25-year review. Ophthalmology. 2014;121(8):1634-1642. 
7. Yao K, Zhu Y, Zhu Z, et al. The incidence of postoperative endophthalmitis after cataract surgery in China: a multicenter investigation of 2006-2011. Br J Ophthalmol. 2013;97(10):1312-1317.

8. Mollan SP, Gao A, Lockwood A, Durrani OM, Butler L. Postcataract endophthalmitis: incidence and microbial isolates in a United Kingdom region from 1996 through 2004. J Cataract Refract Surg. 2007; 33(2):265-268.

9. Kodjikian L, Salvanet-Bouccara A, Grillon S, et al; French Collaborative Study Group on Endophthalmitis. Postcataract acute endophthalmitis in France: national prospective survey. J Cataract Refract Surg. 2009; 35(1):89-97.

10. Olson R, Donnenfeld E, Bucci FA Jr, et al. Methicillin resistance of Staphylococcus species among health care and nonhealth care workers undergoing cataract surgery. Clin Ophthalmol. 2010;4: $1505-1514$.

11. Hsu HY, Lind JT, Tseng L, Miller D. Ocular flora and their antibiotic resistance patterns in the Midwest: a prospective study of patients undergoing cataract surgery. Am J Ophthalmol. 2013;155(1):36.e-44.e.

12. Suto C, Morinaga M, Yagi T, Tsuji C, Toshida H. Conjunctival sac bacterial flora isolated prior to cataract surgery. Infect Drug Resist. 2012; 5:37-41.

13. Endophthalmitis Study Group; European Society of Cataract and Refractive Surgeons. Prophylaxis of postoperative endophthalmitis following cataract surgery: results of the ESCRS multicenter study and identification of risk factors. J Cataract Refract Surg. 2007;33(6):978-988.

14. DeCroos FC, Afshari NA. Perioperative antibiotics and antiinflammatory agents in cataract surgery. Curr Opin Ophthalmol. 2008; 19(1):22-26

15. Chang DF, Braga-Mele R, Mamalis N, et al; ASCRS Cataract Clinical Committee. Prophylaxis of postoperative endophthalmitis after cataract surgery: results of the 2007 ASCRS member survey. J Cataract Refract Surg. 2007;33(10):1801-1805.

16. Rudnisky CJ, Wan D, Weis E. Antibiotic choice for the prophylaxis of post-cataract extraction endophthalmitis. Ophthalmology. 2014;121(4):835-841.

17. Scoper SV. Review of third- and fourth-generation fluoroquinolones in ophthalmology: in vitro and in vivo efficacy. Adv Ther. 2008;25(10):979-994.

18. McDonald M, Blondeau JM. Emerging antibiotic resistance in ocular infections and the role of fluoroquinolones. J Cataract Refract Surg. 2010;36(9):1588-1598.

19. Schimel AM, Miller D, Flynn HW. Endophthalmitis isolates and antibiotic susceptibilities: a 10-year review of culture-proven cases. $\mathrm{Am}$ J Ophthalmol. 2013;156(1):50.e-52.e.

20. Bispo PJ, Alfonso EC, Flynn HW, Miller D. Emerging 8-methoxyfluoroquinolone resistance among methicillin-susceptible Staphylococcus epidermidis isolates recovered from patients with endophthalmitis. J Clin Microbiol. 2013;51(9):2959-2963.

21. Melo GB, Bispo PJ, Yu MC, Pignatari AC, Höfling-Lima AL. Microbial profile and antibiotic susceptibility of culture-positive bacterial endophthalmitis. Eye. 2011;25(3):382-388.

22. Haas W, Pillar CM, Zurenko GE, Lee JC, Brunner LS, Morris TW. Besifloxacin, a novel fluoroquinolone, has broad-spectrum in vitro activity against aerobic and anaerobic bacteria. Antimicrob Agents Chemother. 2009;53(8):3552-3560.

23. Miller D, Chang JS, Flynn HW, Alfonso EC. Comparative in vitro susceptibility of besifloxacin and seven comparators against ciprofloxacinand methicillin-susceptible/nonsusceptible staphylococci. J Ocul Pharmacol Ther. 2013;29(3):339-344.

24. Haas W, Gearinger LS, Usner DW, Decory HH, Morris TW. Integrated analysis of three bacterial conjunctivitis trials of besifloxacin ophthalmic suspension, $0.6 \%$ : etiology of bacterial conjunctivitis and antibacterial susceptibility profile. Clin Ophthalmol. 2011;5:1369-1379.

25. Haas W, Gearinger LS, Hesje CK, Sanfilippo CM, Morris TW. Microbiological etiology and susceptibility of bacterial conjunctivitis isolates from clinical trials with ophthalmic, twice-daily besifloxacin. Adv Ther. 2012;29(5):442-455.
26. Haas W, Pillar CM, Torres M, Morris TW, Sahm DF. Monitoring antibiotic resistance in ocular microorganisms: results from the ARMOR 2009 surveillance study. Am J Ophthalmol. 2011;152(4):567-574.

27. Cambau E, Matrat S, Pan XS, et al. Target specificity of the new fluoroquinolone besifloxacin in Streptococcus pneumoniae, Staphylococcus aureus and Escherichia coli. J Antimicrob Chemother. 2009;63(3): 443-450.

28. MoshirfarM,FeizV, Vitale AT, Wegelin JA, BasavanthappaS, Wolsey DH. Endophthalmitis after uncomplicated cataract surgery with the use of fourth generation fluoroquinolones: a retrospective observational case series. Ophthalmology. 2007;114(4):686-691.

29. Jensen MK, Fiscella RG, Moshirfar M, Mooney B. Third and fourthgeneration fluoroquinolones: retrospective comparison of endophthalmitis after cataract surgery performed over 10 years. J Cataract Refract Surg. 2008;34(9):1460-1467.

30. Clinical and Laboratory Standards Institute. Methods for Dilution Antimicrobial Susceptibility Tests for Bacteria that Grow Aerobically: Approved Standard-Eighth Edition. Wayne, PA: CLSI; 2009. [CLSI document M07-A8].

31. Clinical and Laboratory Standards Institute. Performance Standards for Antimicrobial Susceptibility Testing: Nineteenth Informational Supplement. Wayne, PA: CLSI; 2013. [CLSI document M100-S23].

32. Leibowitz HM. Antibacterial effectiveness of ciprofloxacin $0.3 \%$ ophthalmic solution in the treatment of bacterial conjunctivitis. Am J Ophthalmol. 1991;112(suppl 4):29S-33S.

33. Ta CN, Egbert PR, Singh K, Shriver EM, Blumenkranz MS, Miño De Kaspar H. Prospective randomized comparison of 3-day vs 1-hour preoperative prophylaxis for cataract surgery. Ophthalmology. 2002;109(11):2036-2041.

34. Suzuki T, Tanaka H, Toriyama K, et al. Prospective clinical evaluation of $1.5 \%$ levofloxacin ophthalmic solution in ophthalmic perioperative disinfection. J Ocul Pharmacol Ther. 2013;29(10):887-892.

35. Inoue Y, Usui M, Ohashi Y, Shiota H, Yamazaki T; Preoperative Disinfection Study Group. Preoperative disinfection of the conjunctival sac with antibiotics and iodine compounds: a prospective randomized multicenter study. Jap J Ophthalmol. 2008;52(3):151-161.

36. Snyder-Pelmutter LS, Katz HR, Melia M. Effect of topical ciprofloxacin $0.3 \%$ and ofloxacin $0.3 \%$ on the reduction of bacterial flora on the human conjunctiva. J Cataract Refract Surg. 2000;26(11):1620-1625.

37. Moss JM, Nguyen D, Liu YI, et al. Comparison of one-day versus one-hour application of topical gatifloxacin in eliminating conjunctival bacterial flora. Ophthalmology. 2008;115(11):2013-2016.

38. Haas W, Pillar CM, Hesje CK, Sanfilippo CM, Morris TW. Bactericidal activity of besifloxacin against staphylococci, Streptococcus pneumoniae and Haemophilus influenzae. J Antimicrob Chemother. 2010;65(7):1441-1447.

39. Haas W, Pillar CM, Hesje CK, Sanfilippo CM, Morris TW. In vitro time-kill experiments with besifloxacin, moxifloxacin and gatifloxacin in the absence and presence of benzalkonium chloride. $J$ Antimicrob Chemother. 2011;66(4):840-844.

40. Bowman LM, Si E, Pang J, Archibald R, Friedlaender M. Development of a topical polymeric mucoadhesive ocular delivery system for azithromycin. J Ocul Pharmacol Ther. 2009;25(2):133-139.

41. Akpek EK, Vittitow J, Verhoeven RS, et al. Ocular surface distribution and pharmacokinetics of a novel ophthalmic 1\% azithromycin formulation. J Ocul Pharmacol Ther. 2009;25(5):433-439.

42. Si EC, Bowman LM, Hosseini K. Pharmacokinetic comparisons of bromfenac in DuraSite and Xibrom. J Ocul Pharmacol Ther. 2011; 27(1):61-66.

43. Shafiee A, Bowman LM, Hou E, Hosseini K. Aqueous humor penetration of ketorolac formulated in DuraSite or DuraSite 2 delivery systems compared to Acular LS in rabbits. J Ocul Pharmacol Ther. 2013;29(9):812-816.

44. Shafiee A, Bowman LM, Hou E, Hosseini K. Ocular pharmacokinetics of bimatoprost formulated in DuraSite compared to bimatoprost $0.03 \%$ ophthalmic solution in pigmented rabbit eyes. Clin Ophthalmol. 2013; $7: 1549-1556$. 
45. Miller D, Flynn PM, Scott IU, Alfonso EC, Flynn HW Jr. In vitro fluoroquinolone resistance in staphylococcal endophthalmitis isolates. Arch Ophthalmol. 2006;124(4):479-483.

46. Deramo VA, Lai JC, Fastenberg DM, Udell IJ. Acute endophthalmitis in eyes treated prophylactically with gatifloxacin and moxifloxacin. Am J Ophthalmol. 2006;142(5):721-725.

47. Deramo VA, Lai JC, Winokur J, Luchs J, Udell IJ. Visual outcome and bacterial sensitivity after methicillin-resistant Staphylococcus aureus-associated acute endophthalmitis. Am J Ophthalmol. 2008; 145(3):413-417.

48. Recchia FM, Busbee BG, Pearlman RB, Carvalho-Recchia CA, Ho AC. Changing trends in the microbiologic aspects of post-cataract endophthalmitis. Arch Ophthalmol. 2005;123(3):341-346.

49. Major JC Jr, Engelbert M, Flynn HW Jr, Miller D, Smiddy WE, Davis JL. Staphylococcus aureus endophthalmitis: antibiotic susceptibilities, methicillin resistance, and clinical outcomes. Am JOphthalmol. 2010;149(2):278-283

50. Malhotra R, Gira J, Berdy GJ, Brusatti R. Safety and tolerance of besifloxacin ophthalmic suspension $0.6 \%$ as a prophylactic antibiotic following routine cataract surgery: results of a prospective, parallelgroup investigator-masked study. Clin Ophthalmol. 2012;6:855-863.
51. Parekh JG, Newsom TH, Nielsen S. Safety of besifloxacin ophthalmic suspension $0.6 \%$ in cataract surgery patients. J Cataract Refract Surg. 2012; 38(10):1864-1867

52. Majmudar PA, Clinch TE. Safety of besifloxacin ophthalmic suspension $0.6 \%$ in cataract or LASIK surgery patients. Cornea. 2014;33(5): $457-462$.

53. Nielsen SA, McDonald MB, Majmudar PA. Safety of besifloxacin ophthalmic suspension $0.6 \%$ in refractive surgery: a retrospective chart review of post-LASIK patients. [corrigendum in Clin Ophthalmol. 2013;7:725]. Clin Ophthalmol. 2013;7:149-156.

54. Donnenfeld E, Lane S, Holland E, et al. A prospective, contralateral eye, double-masked study of the effect of besifloxacin $0.6 \%$ and moxifloxacin $0.5 \%$ on corneal epithelial wound healing following photorefractive keratectomy. Poster presented at: ASCRS Symposium on Cataract, IOL and Refractive Surgery; April 2013; San Francisco, CA.

55. Blondeau JM, Borsos S, Hesje CK. Antimicrobial efficacy of gatifloxacin and moxifloxacin with and without benzalkonium chloride compared with ciprofloxacin and levofloxacin against methicillin-resistant Staphylococcus aureus. J Chemother. 2007;19(2):146-151.
Clinical Ophthalmology

\section{Publish your work in this journal}

Clinical Ophthalmology is an international, peer-reviewed journal covering all subspecialties within ophthalmology. Key topics include: Optometry; Visual science; Pharmacology and drug therapy in eye diseases; Basic Sciences; Primary and Secondary eye care; Patient Safety and Quality of Care Improvements. This journal is indexed on

\footnotetext{
Submit your manuscript here: http://www.dovepress.com/clinical-ophthalmology-journal
}

\section{Dovepress}

PubMed Central and CAS, and is the official journal of The Society of Clinical Ophthalmology (SCO). The manuscript management system is completely online and includes a very quick and fair peer-review system, which is all easy to use. Visit http://www.dovepress.com/ testimonials.php to read real quotes from published authors. 